\title{
CONSTRUCTIVE INTERFERENCE EXPLOITATION FOR DOWNLINK BEAMFORMING BASED ON NOISE ROBUSTNESS AND OUTAGE PROBABILITY SPECIFICATIONS RESPECTIVELY
}

\author{
Ka Lung Law, Christos Masouros \\ Department of Electrical and Electronic Engineering, University College London, London, UK \\ E-mail: k.law@ucl.ac.uk, chris.masouros@ieee.org
}

\begin{abstract}
Quality of service (QoS) is commonly measured in terms of signal to interference plus noise ratio (SINR), where multiuser interference is mitigated in order to improves the performance. Despite of suppressing, interference can be exploited constructively to enhance the desired signal. With the aid of channel state information (CSI) at the transmitter and data information, we propose symbol-level downlink beamforming problems based on noise robustness and outage probability specifications, respectively, subject to power constraints. We show that an equivalence relationship between the noise robustness and outage probability symbol-level downlink beamforming problems is obtained. Finally, we provide an analytic symbol error rate (SER) upper bound of the worst user by solving the outage probability-based problem. Our simulations demonstrate that the proposed techniques provide substantial performance improvements over conventional downlink beamforming techniques.
\end{abstract}

Index Terms - Downlink beamforming, robust design, error probability, convex optimization, constructive interference.

\section{INTRODUCTION}

In wireless networks, downlink beamforming is an attractive approach due to an effective way of simultaneously transmitting an individual data for each user to achieve demand in high data rate. [1,2]. In addition to the urge for high throughputs and limited power expenses, quality of service (QoS) is also a main criteria in modern communications systems. With the knowledge of channel state information (CSI) at the transmitter, designing downlink beamformers to improve the QoS for downlink scenario has been studied extensively [3-9].

Zero-forcing (ZF) precoding is commonly employed to downlink problem. The multiuser interference signals is nulled in wireless communications [10,11]. The advantage of ZF precoding is that the algorithm is simple to apply. However, it is not fully optimized. To obtain the optimal solutions, the optimization-based downlink beamforming problems were developed [4, 5, 12,-15]. One form of downlink problems is to maximize the minimum SINR subject to a total power constraint [4]. The problem is efficiently solved using an iterative algorithm. Taking the CSI mismatch into account, channel robust worst-case downlink beamforming optimization was considered [5, 12, 14]. To provide more flexibility than the worst-case scenario, channel outage probability-based downlink beamforming optimization has been introduced [14, 15]. It has been proved that both the worst channel robustness and outage probability-based problems are equivalent.

In the SINR-based downlink problem, beamformers are designed to guarantee that the SINR constraints are satisfied. However, the drawback of SINR criteria is that power is wasted by suppressing the interference. Rather than mitigating, one can exploit constructive interference to enhance the useful signal by making use of both the CSI and data information. By exploiting the constructive interference to achieve higher performance, the closed-form linear and non-linear precoders were discussed [16-|22]. Nonetheless, these precoders are not the optimal design. Optimization-based downlink beamforming precoders by exploiting constructive interference was considered [23, 24].

In line with the above, this paper is based on the symbol-level downlink beamforming optimization by exploiting constructive interference to amplify the signal [23, 24]. In the following analysis, phase-shift keying (PSK) modulation is selected. We assume that a TDD transmission, e.g., downlink channels can be determined by using the knowledge of uplink CSI and uplink-downlink channel reciprocity [25], the availability of perfect CSI at the transmitter and instantaneous data information, as in [23, 24]. We propose a symbol-level downlink beamforming problem based on noise robust design in Section 4 by introducing a geometrical analysis to the optimization problem studied in [23]. We reformulate the optimization to address the symbol-level downlink beamforming problem based on outage probability design in Section 5 by use of duality with the noise robust case. All proposed approaches can be formulated into convex optimizations and can be solved efficiently. We provide an analytic symbol error rate (SER) upper bound of the worst user by solving the error probability-based optimization.

Notation: $\mathrm{E}(\cdot), \operatorname{Pr}(\cdot),|\cdot|,\|\cdot\|,(\cdot)^{*}(\cdot)^{T}$, denote statistical expectation, the probability, the absolute value, the Euclidean norm, the complex conjugate, the transpose, respectively. $\operatorname{Re}(\cdot)$ and $\operatorname{Im}(\cdot)$ are the real part, and the imaginary part, respectively.

\section{SYSTEM MODEL AND CONVENTIONAL DOWNLINK BEAMFORMING}

Let us consider a downlink scenario with a single $N$-antenna at the BS and transmits signals. We assume that there are $K$ single-antenna users. Let $b_{i}$ be the transmitted data with the unit amplitude of the $M$-order PSK modulation and the given maximum angular shift $\theta=$ $\pi / M$. The transmitted signal at the $\mathrm{BS}$ is the $N \times 1$ vector

$$
\mathbf{x}=\sum_{i=1}^{K} \mathbf{t}_{i} b_{i}
$$

where $\mathbf{h}_{i}, \mathbf{t}_{i}$, and $\sigma^{2}$ are the $N \times 1$ transmitted signal vector for the $i$ th user, the $N \times 1$ beamforming vector for the $i$ th user, and the noise variance, respectively. The received signal for the $i$ th user is given 


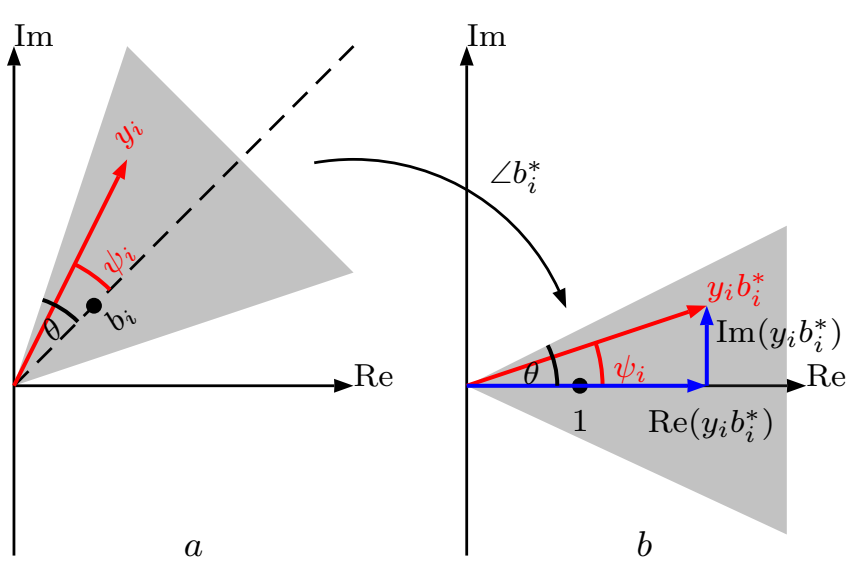

Fig. 1: In $M$-PSK, a) constructive interference $y_{i}$ within correct detection region; b) vector decomposition of $y_{i} b_{i}^{*}$ after rotation by $\angle b_{i}^{*}$.

by

$$
y_{i}=\mathbf{h}_{i}^{T} \mathbf{x}+n_{i}
$$

We present a common downlink beamforming optimization problem in the literature [4,6], which maximizes to the minimum SINR subject to a total transmitted power constraint. The problem can be formulated as [4]

$$
\begin{aligned}
\max _{\mathbf{t}_{i}, \gamma} & \gamma \\
\text { s.t. } & \frac{\left|\mathbf{h}_{i}^{T} \mathbf{t}_{i}\right|^{2}}{\sum_{\substack{j=1 \\
j \neq i}}^{K}\left|\mathbf{h}_{j}^{T} \mathbf{t}_{j}\right|^{2}+\sigma^{2}} \geq \gamma, \forall i=1, \ldots, K, \\
& \sum_{i=1}^{K}\left\|\mathbf{t}_{i}\right\|^{2} \leq P_{0},
\end{aligned}
$$

where $P_{0}$ is the given total transmitted power threshold.

\section{CONSTRUCTIVE INTERFERENCE OPTIMIZATION-BASED PRECODING}

By jointly exploiting the knowledge of the CSI and user data information at the transmitter, the constructive interference-based optimization precoder in [23] improves upon the above conventional optimization. The precoder maximizes the shifted distance (SD) of correct detection region away from origin along with the direction of the corresponding transmitted symbol $b_{i}$ by designing the beamformers. The optimal beamformers can guarantee that the resultant received symbol $\mathbf{h}_{i}^{T} \mathbf{x}$ still falls within the corresponding region. Under the design criteria, the resultant received symbol moves away from the original decision thresholds of the constellation. This leads to improve the QoS. The reader interested in additional details of the underlying concept is referred to [23]. The optimization problem can be written in mathematical form as [23]

$$
\begin{aligned}
\max _{\mathbf{x}, \tau} & \tau \\
\text { s.t. } & \left|\operatorname{Im}\left(b_{i}^{*} \mathbf{h}_{i}^{T} \mathbf{x}\right)\right| \leq\left(\operatorname{Re}\left(b_{i}^{*} \mathbf{h}_{i}^{T} \mathbf{x}\right)-\tau \sigma\right) \tan \theta, \\
& \|\mathbf{x}\|^{2} \leq P_{0}, \forall i=1, \ldots, K,
\end{aligned}
$$

where $P_{0}$ is the predefined total transmitted power threshold. The constraints of (4) stem from the fact that the resultant received symbol for the $i$ th user lays on correct detection region, if and only if $-\theta \leq \phi_{i} \leq \theta$ where $\phi_{i}$ is an angle such that

$$
\phi_{i}(\mathbf{x}, \tau)= \begin{cases}\tan ^{-1}\left(\frac{\operatorname{Im}\left(b_{i}^{*} \mathbf{h}_{i}^{T} \mathbf{x}\right)}{\operatorname{Re}\left(b_{i}^{*} \mathbf{h}_{i}^{T} \mathbf{x}\right)-\tau \sigma}\right) & \operatorname{Re}\left(b_{i}^{*} \mathbf{h}_{i}^{T} \mathbf{x}\right)>\tau \sigma, \\ 0 & b_{i}^{*} \mathbf{h}_{i}^{T} \mathbf{x}=\tau \sigma .\end{cases}
$$

The disadvantage of (4) are that it is hard to quantify the QoS in terms of the SD. In particular, [23] did not provide the relationship between the SD and the worst user's SER performance. We address this issue in Section 5 In the next section we present a noise robustness-based optimization by exploiting the constructive interference.

\section{NOISE ROBUST BEAMFORMING OPTIMIZATION}

In this section, we introduce a noise robust adaptation together with exploiting the constructive interference. First of all, we present an improved systematic treatment of constructive interference for the received signal. For PSK modulation, interference is constructive 1 if the received signal $y_{i}$ lays on the correct detection region, which is the shaded area shown in Fig. 1 (a). Under the definition of constructive interference, we obtain the following lemma.

Lemma 1. The received signal $y_{i}$ is said to receive constructive interference, if and only if

$$
-\theta \leq \psi_{i} \leq \theta
$$

where $\psi_{i}$ in Fig. 1 (a) is the angle between the received signal $y_{i}$ and the transmitted symbol $b_{i}$ such that

$$
\psi_{i}\left(\mathbf{x}, n_{i}\right)= \begin{cases}\tan ^{-1}\left(\frac{\operatorname{Im}\left(y_{i} b_{i}^{*}\right)}{\operatorname{Re}\left(y_{i} b_{i}^{*}\right)}\right) & \operatorname{Re}\left(y_{i} b_{i}^{*}\right)>0 \\ 0 & y_{i} b_{i}^{*}=0\end{cases}
$$

The criteria in (6) can be directly reformulated as the following constraints

$$
\left|\operatorname{Im}\left(y_{i} b_{i}^{*}\right)\right|-\operatorname{Re}\left(y_{i} b_{i}^{*}\right) \tan \theta \leq 0 .
$$

Proof. Suppose that the received signal $y_{i}$ is within the correct detection region. To obtain the angle $\psi_{i}$, we first rotate Fig. 1 (a) to Fig. 1. (b) by shifting the constellation by a phase equal to $\angle b_{i}^{*}$, i.e., by multiplying $b_{i}^{*}$. As $b_{i}$ is a unit power, $y_{i} b_{i}^{*}$ does not change the magnitude. Then we obtain the inequities in (7) where $\operatorname{Im}\left(y_{i} b_{i}^{*}\right)$ and $\operatorname{Re}\left(y_{i} b_{i}^{*}\right)$ are the projection of $y_{i} b_{i}^{*}$ onto the real and imaginary axis, respectively.

\subsection{Noise Uncertainty Radius Maximization}

The idea of the symbol-level downlink beamforming problem based on noise robustness specification is to design the beamformers such that the received signal is constructive interference if the noise is within the noise uncertainty set. To improve the noise robustness of the design given the noise variance $\sigma^{2}$, we maximize the radius of the noise uncertainty set such that it can still satisfy the constraints 8

\footnotetext{
${ }^{1}$ Note that we consider the resultant received symbol plus noise in our case, while [23] discussed the resultant received symbol $\mathbf{h}_{i}^{T} \mathbf{x}$ in the formulation.
} 
under the power constraint. The noise robustness-based optimization problem by exploiting constructive interference can be written as

$$
\begin{gathered}
\max _{\mathbf{x}, \Gamma} \Gamma \text { s.t. } \max _{\left\|n_{i}\right\| \leq \Gamma \sigma}\left|\psi_{i}\left(\mathbf{x}, n_{i}\right)\right| \leq \theta, \forall i=1, \ldots, K, \\
\|\mathbf{x}\|^{2} \leq P,
\end{gathered}
$$

where $P$ is the given total transmit power. By Lemma 1, we rewrite (9) as

$$
\begin{gathered}
\max _{\mathbf{x}, \Gamma} \Gamma \text { s.t. } \max _{\left\|n_{i}\right\| \leq \Gamma \sigma}\left|\operatorname{Im}\left(y_{i} b_{i}^{*}\right)\right|-\operatorname{Re}\left(y_{i} b_{i}^{*}\right) \tan \theta \leq 0, \\
\|\mathbf{x}\|^{2} \leq P, \forall i=1, \ldots, K
\end{gathered}
$$

To simplify above problem, we can first solve the inner maximization in (10).

Corollary 1. For a fixed $\tilde{\mathbf{x}}$, the inner maximization in (10) has the following optimal solution as

$$
\left|\operatorname{Im}\left(b_{i}^{*} \mathbf{h}_{i}^{T} \tilde{\mathbf{x}}\right)\right|+\Gamma \sigma / \cos \theta-\operatorname{Re}\left(b_{i}^{*} \mathbf{h}_{i}^{T} \tilde{\mathbf{x}}\right) \tan \theta .
$$

Proof. Let $\tilde{y}_{i}=b_{i}^{*} \mathbf{h}_{i}^{T} \tilde{\mathbf{x}}+n_{i}$. The dual Lagrange function is given by

$$
\mathcal{L}\left(\kappa_{i}, n_{i}\right)=-\left|\operatorname{Im}\left(\tilde{y}_{i}\right)\right|+\operatorname{Re}\left(\tilde{y}_{i}\right) \tan \theta+\kappa_{i}\left(\left\|n_{i}\right\|^{2}-\Gamma^{2} \sigma^{2}\right),
$$

where $\kappa_{i} \geq 0$. Note that

$$
\begin{aligned}
& \operatorname{Im}\left(b_{i}^{*} n_{i}\right)=n_{I i} b_{R i}-n_{R i} b_{I i}, \\
& \operatorname{Re}\left(b_{i}^{*} n_{i}\right)=n_{I i} b_{I i}+n_{R i} b_{R i} .
\end{aligned}
$$

where $n_{i} \triangleq n_{R i}+i n_{I i}$, and $b_{i} \triangleq b_{R i}+i b_{I i}$. Setting $\frac{\partial \mathcal{L}}{\partial n_{R i}}=0$ and $\frac{\partial \mathcal{L}}{\partial n_{I i}}=0$, we obtain

$$
\begin{aligned}
b_{R i} \tan \theta+b_{I i} \alpha_{i}+2 \kappa_{i}^{\star} n_{R i}^{\star} & =0, \\
-b_{R i} \alpha_{i}+b_{I i} \tan \theta+2 \kappa_{i}^{\star} n_{I i}^{\star} & =0,
\end{aligned}
$$

where $\alpha_{i}=\operatorname{Im}\left(\tilde{y}_{i}\right) /\left|\operatorname{Im}\left(\tilde{y}_{i}\right)\right|$ and $a^{\star}$ is the optimal value of $a$. If we suppose that $\kappa_{i}^{\star}=0$, then (15) implies that $b_{R i}=b_{I i}=0$, which leads to the contradiction. Therefore, we conclude that $\kappa_{i}^{\star}>0$ and

$$
\left\|n_{i}^{\star}\right\|^{2}=\Gamma^{2} \sigma^{2},
$$

by the complementary slackness. Putting 15 into 16 and the fact that $b_{i}$ is an unit power symbol, we obtain

$$
\kappa_{i}^{\star}=(2 \Gamma \sigma \cos \theta)^{-1} .
$$

We substitute (17) back into (15), then we get

$$
\begin{aligned}
& n_{R i}^{\star}=-\left(b_{R i} \tan \theta+b_{I i} \alpha_{i}\right) \Gamma \sigma \cos \theta, \\
& n_{R i}^{\star}=\left(b_{R i} \alpha_{i}-b_{I i} \tan \theta\right) \Gamma \sigma \cos \theta .
\end{aligned}
$$

Taking (18) into problem (10), we rewrite the inner maximization in (10) as

$$
\begin{aligned}
& \alpha_{i} \operatorname{Im}\left(b_{i}^{*} \mathbf{h}_{i}^{T} \tilde{\mathbf{x}}\right)-n_{R i}^{\star}\left(b_{R i} \tan \theta+b_{I i} \alpha_{i}\right) \\
& +n_{I i}^{\star}\left(b_{R i} \alpha_{i}-b_{I i} \tan \theta\right)-\operatorname{Re}\left(b_{i}^{*} \mathbf{h}_{i}^{T} \tilde{\mathbf{x}}\right) \tan \theta \\
& =\left|\operatorname{Im}\left(b_{i}^{*} \mathbf{h}_{i}^{T} \tilde{\mathbf{x}}\right)\right|+\Gamma \sigma / \cos \theta-\operatorname{Re}\left(b_{i}^{*} \mathbf{h}_{i}^{T} \tilde{\mathbf{x}}\right) \tan \theta
\end{aligned}
$$

where $\operatorname{Im}\left(\tilde{y}_{i}\right)$ and $\operatorname{Re}\left(b_{i}^{*} \mathbf{h}_{i}^{T} \tilde{\mathbf{x}}\right)$ have the same sign because we can assume that the received noise cannot dominate the received signal.
According to Corollary 1, we reformulate (10) as a function $\Gamma^{\star}(\cdot)$ for any given $P \geq 0$ such that

$$
\begin{aligned}
\Gamma^{\star}(P): \max _{\mathbf{x}, \Gamma} & \Gamma \\
\text { s.t. } & \left|\operatorname{Im}\left(b_{i}^{*} \mathbf{h}_{i}^{T} \mathbf{x}\right)\right|+\Gamma \sigma / \cos \theta \leq \operatorname{Re}\left(b_{i}^{*} \mathbf{h}_{i}^{T} \mathbf{x}\right) \tan \theta, \\
& \|\mathbf{x}\|^{2} \leq P, \forall i=1, \ldots, K .
\end{aligned}
$$

Problem 20 can be solved using available convex optimization tools [26]. Finally, we obtain the optimal beamformer $\mathbf{t}_{i}^{\star}$ in (1) as

$$
\mathbf{t}_{i}^{\star}=\mathbf{x}^{\star} b_{i}^{*} / K,
$$

where $\mathrm{x}^{\star}$ is the optimal solution in (20).

Remark: Suppose $\mathbf{x}_{S D}^{\star}$ and $\mathbf{x}_{N R}^{\star}$ are optimal solutions of (4) and (20), respectively. Then $\sin \theta \mathbf{x}_{N R}^{\star}=\mathbf{x}_{S D}^{\star}$. Hence we can treat them as equivalence problems.

\section{OUTAGE PROBABILITY APPROACH}

We assume a noise at the receiver is complex Gaussian with zero mean. In this section, we present a new approach to constructive interference-based downlink beamforming by the noise outage probability. In the concept of noise outage probability, we replace the noise robust downlink beamforming constraints by more flexible probabilistic constraints. We define the noise outage probability for the $i$ th constraint as the probability that received signal lays outside the correct detection region bounded by either the angle $\theta$ or $-\theta$. The problem can be written as

$$
\begin{aligned}
& \min _{\mathbf{x}, p} p \text { s.t. } \operatorname{Pr}\left(\pi \geq \psi_{i}\left(\mathbf{x}, n_{i}\right) \geq \theta\right) \leq p, \forall i=1, \ldots, K \\
& \operatorname{Pr}\left(-\pi \geq \psi_{i}\left(\mathbf{x}, n_{i}\right) \geq-\theta\right) \leq p, \forall i=1, \ldots, K \\
&\|\mathbf{x}\|^{2} \leq P .
\end{aligned}
$$

Remark: Problem (22) and the channel outage probability based downlink beamforming problem in [14,15] are different. The constraints in [14, 15] are outage probabilistic SINR-based with channel random variables, while the constraints are outage probabilistic constructive interference-based with noise random variables. The SER upper bound of the worst user is equal to $2 p$, which is originated from that the worst case possibility of the received signal laying oustide the correct detection region bounded by the angle $\pm \theta$ is $p$. It will be shown in the simulation result that the worst user's SER performance calculations close to the upper bound.

According to Lemma 1, problem (22) can be expressed as

$$
\begin{aligned}
& \min _{\mathbf{x}, p} p \text { s.t. } \operatorname{Pr}\left(\operatorname{Im}\left(y_{i} b_{i}^{*}\right) \geq \operatorname{Re}\left(y_{i} b_{i}^{*}\right) \tan \theta\right) \leq p, \\
& \operatorname{Pr}\left(\operatorname{Im}\left(y_{i} b_{i}^{*}\right) \leq-\operatorname{Re}\left(y_{i} b_{i}^{*}\right) \tan \theta\right) \leq p, \\
&\|\mathbf{x}\|^{2} \leq P, \forall i=1, \ldots, K .
\end{aligned}
$$

The constraints in (23a) and $23 \mathrm{~b}$ ) can be rewritten as

$$
\operatorname{Pr}\left(z_{i}+\tilde{n}_{i} \geq 0\right) \leq p,
$$

where

$$
\begin{aligned}
z_{i} & = \pm \operatorname{Im}\left(b_{i}^{*} \mathbf{h}_{i}^{T} \mathbf{x}\right)-\operatorname{Re}\left(b_{i}^{*} \mathbf{h}_{i}^{T} \mathbf{x}\right) \tan \theta, \\
\tilde{n}_{i} & = \pm \operatorname{Im}\left(b_{i}^{*} n_{i}\right)-\operatorname{Re}\left(b_{i}^{*} n_{i}\right) \tan \theta .
\end{aligned}
$$




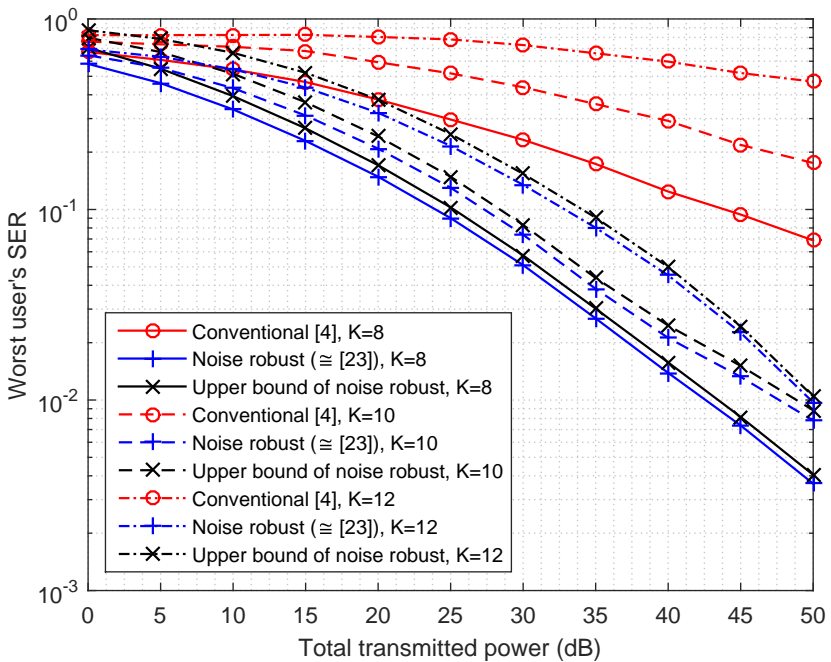

Fig. 2: The worst user's SER performance versus transmit power with $N=10$.

As $n_{i}$ is complex Gaussian, we obtain

$$
\begin{aligned}
& \mathrm{E}\left\{\operatorname{Re}\left(b_{i}^{*} n_{i}\right)^{2}\right\}=\mathrm{E}\left\{\operatorname{Im}\left(b_{i}^{*} n_{i}\right)^{2}\right\}=b_{R i}^{2} \frac{\sigma^{2}}{2}+b_{I i}^{2} \frac{\sigma^{2}}{2}=\frac{\sigma^{2}}{2}, \\
& \mathrm{E}\left\{\operatorname{Re}\left(b_{i}^{*} n_{i}\right) \operatorname{Im}\left(b_{i}^{*} n_{i}\right)\right\}=b_{R i} b_{I i}-b_{R i} b_{I i}=0 .
\end{aligned}
$$

The variance of $n_{i}$ is given by

$$
\mathrm{E}\left\{\tilde{n}_{i}^{2}\right\}=\left(1+\tan ^{2} \theta\right) \sigma^{2} / 2=\sigma^{2} /\left(2 \cos ^{2} \theta\right) .
$$

Therefore, $\tilde{n}_{i} \sim \mathcal{N}\left(0, \frac{\sigma}{\sqrt{2} \cos \theta}\right)$. By ensuring reliable communication link, the noise outage probability must be close to 0 . According to [15], we assume that $p \leq 0.5$. The outage probability constraints in (24) can be expressed in terms of the Gaussian error function $\operatorname{erf}(\cdot)$ as

$$
\frac{1}{2}-\frac{1}{2} \operatorname{erf}\left(\frac{-z_{i} \cos \theta}{\sigma}\right) \leq p,
$$

or equivalently,

$$
\left|\operatorname{Im}\left(b_{i}^{*} \mathbf{h}_{i}^{T} \mathbf{x}\right)\right|+\frac{\operatorname{erf}^{-1}(1-2 p) \sigma}{\cos \theta} \leq \operatorname{Re}\left(b_{i}^{*} \mathbf{h}_{i}^{T} \mathbf{x}\right) \tan \theta, \forall i
$$

Hence, the outage probability problem (23) can be written as a function $p^{\star}(\cdot)$ for any given $P \geq 0$ such that

$$
\begin{array}{rl}
p^{\star}(P): \min _{\mathbf{x}, p} & p \\
\text { s.t. } & \operatorname{Im}\left(b_{i}^{*} \mathbf{h}_{i}^{T} \mathbf{x}\right) \mid+\frac{\operatorname{erf}^{-1}(1-2 p) \sigma}{\cos \theta} \leq \operatorname{Re}\left(b_{i}^{*} \mathbf{h}_{i}^{T} \mathbf{x}\right) \tan \theta \\
& \|\mathbf{x}\|^{2} \leq P, \forall i=1, \ldots, K .
\end{array}
$$

and the optimal values of (20) and (32) have the following relations:

$$
\begin{aligned}
\Gamma^{\star}(P) & =\operatorname{erf}^{-1}\left(1-2 p^{\star}(P)\right), \\
p^{\star}(P) & =\frac{1}{2}-\frac{1}{2} \operatorname{erf}\left(\Gamma^{\star}(P)\right), \\
\mathbf{x}_{p}^{\star}(P) & =\mathbf{x}_{\Gamma}^{\star}(P),
\end{aligned}
$$

where $\mathbf{x}_{p}^{\star}(\tilde{P})$ is an optimal solution of $(32)$ for a given power $\tilde{P}$.
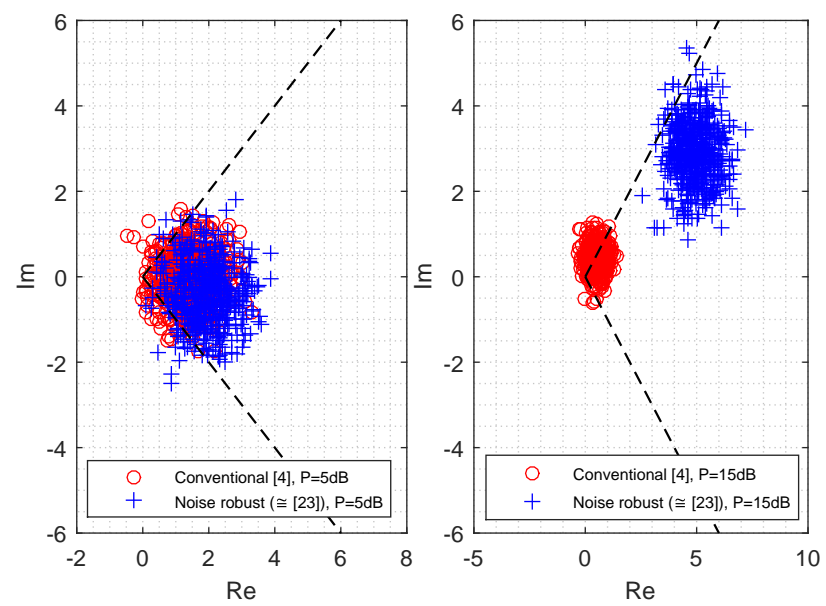

Fig. 3: Distribution of received signals on complex plane with $N=$ 10 , and $K=10$.

\section{SIMULATIONS}

In our simulations, the system with 4-PSK modulation is considered, i.e., $\theta=\pi / 4$, while it is intuitive that the benefits of the proposed approaches extend to other modulation schemes. The white complex zero-mean Gaussian noise $n_{i}$ is with the variance $\sigma^{2}=1$. We consider a constructive interference-based downlink beamforming network with $N=10$ antennas, while it is obvious that the benefits shown extend to different numbers of antennas. Let $\omega_{i}$ be a uniformly distributed random number between $-\pi / 2$ and $\pi / 2$. We model the downlink channel between the BS and $i$ th user as [27]

$$
\mathbf{h}_{i}=\left[1, e^{j \pi \sin \omega_{i}}, \ldots, e^{j \pi(N-1) \sin \omega_{i}}\right]^{T} .
$$

We compare two different techniques: 'Conventional [4]' refers to the SINR balancing problem in [4]; 'Noise robust ( $\cong[2]$ )' stands for the problem (20). Note that (20) is equivalent to (4), which is proposed in [23]. 'Upper bound of noise robust' stands for the SER upper bound of the worst user by solving noise robust approach and it is equal to $2 p$, where $p$ is the outage probability of (32). Since we have shown in Section 5 that the noise robust approach of 20 . and the outage probability approach of 32 are equivalent, we only consider the noise robust approach in the following simulations.

Fig. 2 compare the SER performance for the different techniques. In Fig. 22 we fix the number of users and compare the SER performance of our proposed approaches and the conventional approach of [4] versus the total transmitted power $P$ for $K=8,10,12$. It can be seen from the figure that the noise robust approach outperforms the conventional method of (3). Furthermore, the worst user's SER performance calculations of the proposed noise robust approach match close to the SER upper bound.

Fig. 3 displays the distribution of the received signals using the two techniques on complex plane with $P=5 \mathrm{~dB}$ and $P=15 \mathrm{~dB}$. Here, we set the transmitted symbol to be 1 . The right side of dotted line is the constructive area of the constellation. Therefore, the received signals are valid if they lay on the right side behind the dotted line. We observe from Fig. 3 that the symbols of our proposed method can better lay on the correct detective region compared to the conventional method. Moreover, We notice that when the power increases, our technique can shift the symbols further away from the decision threshold than the conventional technique. 


\section{REFERENCES}

[1] E. Dahlman, S. Parkvall, and J. Skold, 4G: LTE/LTE-Advanced for Mobile Broadband: LTE/LTE-Advanced for Mobile Broadband, Elsevier Science, 2011.

[2] A. F. Molisch, Wireless communications, John Wiley and Sons Ltd, 2011.

[3] F. Rashid-Farrokhi, K.J.R. Liu, and L. Tassiulas, "Transmit beamforming and power control for cellular wireless systems," Selected Areas in Communications, IEEE Journal on, vol. 16, no. 8, pp. 1437-1450, Oct 1998.

[4] M. Schubert and H. Boche, "Solution of the multiuser downlink beamforming problem with individual SINR constraints," IEEE Transactions on Vehicular Technology, vol. 53, no. 1, pp. 18-28, Jan. 2004.

[5] M. Bengtsson and B. Ottersten, "Optimal downlink beamforming using semidefinite optimization," in Annual Allerton Conference on Communication, Control and Computing, 1999, vol. 37, pp. 987-996.

[6] Mats Bengtsson and Björn Ottersten, "Optimal and suboptimal transmit beamforming," Handbook of Antennas in Wireless Communications, 2001.

[7] A Wiesel, Y. C. Eldar, and S. Shamai, "Linear precoding via conic optimization for fixed MIMO receivers," IEEE Transactions on Signal Processing, vol. 54, no. 1, pp. 161-176, Jan. 2006.

[8] A. B. Gershman, N. D. Sidiropoulos, S. Shahbazpanahi, M. Bengtsson, and B. Ottersten, "Convex optimization-based beamforming: From receive to transmit and network designs," IEEE Signal Processing Magazine, vol. 27, no. 3, pp. 62-75, May 2010.

[9] Jinho Choi, "Downlink multiuser beamforming with compensation of channel reciprocity from RF impairments," Communications, IEEE Transactions on, vol. 63, no. 6, pp. 21582169, June 2015.

[10] Q.H. Spencer, A.L. Swindlehurst, and M. Haardt, "Zeroforcing methods for downlink spatial multiplexing in multiuser MIMO channels," Signal Processing, IEEE Transactions on, vol. 52, no. 2, pp. 461-471, Feb 2004.

[11] Kai-Kit Wong, R.D. Murch, and K.B. Letaief, "A joint-channel diagonalization for multiuser mimo antenna systems," Wireless Communications, IEEE Transactions on, vol. 2, no. 4, pp. 773786, 2003.

[12] M.B. Shenouda and T.N. Davidson, "Convex conic formulations of robust downlink precoder designs with quality of service constraints," Selected Topics in Signal Processing, IEEE Journal of, vol. 1, no. 4, pp. 714-724, Dec 2007.

[13] N. Vucic and H. Boche, "Robust QoS-constrained optimization of downlink multiuser MISO systems," Signal Processing, IEEE Transactions on, vol. 57, no. 2, pp. 714-725, Feb 2009.

[14] I. Wajid, M. Pesavento, Y.C. Eldar, and D. Ciochina, "Robust downlink beamforming with partial channel state information for conventional and cognitive radio networks," Signal Processing, IEEE Transactions on, vol. 61, no. 14, pp. 3656-3670, July 2013.

[15] B.K. Chalise, S. ShahbazPanahi, A. Czylwik, and A.B. Gershman, "Robust downlink beamforming based on outage probability specifications," Wireless Communications, IEEE Transactions on, vol. 6, no. 10, pp. 3498-3503, October 2007.
[16] C. Masouros and E. Alsusa, "Soft linear precoding for the downlink of ds/cdma communication systems," Vehicular Technology, IEEE Transactions on, vol. 59, no. 1, pp. 203-215, Jan 2010.

[17] C. Masouros and E. Alsusa, "Dynamic linear precoding for the exploitation of known interference in MIMO broadcast systems," Wireless Communications, IEEE Transactions on, vol. 8, no. 3, pp. 1396-1404, March 2009.

[18] C. Masouros, "Correlation rotation linear precoding for MIMO broadcast communications," Signal Processing, IEEE Transactions on, vol. 59, no. 1, pp. 252-262, Jan 2011.

[19] C. Masouros and T. Ratnarajah, "Interference as a source of green signal power in cognitive relay assisted co-existing MIMO wireless transmissions," Communications, IEEE Transactions on, vol. 60, no. 2, pp. 525-536, February 2012.

[20] C. Masouros, M. Sellathurai, and T. Ratnarajah, "Interference optimization for transmit power reduction in TomlinsonHarashima precoded MIMO downlinks," Signal Processing, IEEE Transactions on, vol. 60, no. 5, pp. 2470-2481, May 2012.

[21] C. Masouros, T. Ratnarajah, M. Sellathurai, C.B. Papadias, and A.K. Shukla, "Known interference in the cellular downlink: a performance limiting factor or a source of green signal power?," Communications Magazine, IEEE, vol. 51, no. 10, pp. 162-171, October 2013.

[22] Gan Zheng, I. Krikidis, C. Masouros, S. Timotheou, D.-A. Toumpakaris, and Zhiguo Ding, "Rethinking the role of interference in wireless networks," Communications Magazine, IEEE, vol. 52, no. 11, pp. 152-158, Nov 2014.

[23] C. Masouros and G. Zheng, "Exploiting known interference as green signal power for downlink beamforming optimization," Signal Processing, IEEE Transactions on, vol. 63, no. 14, pp. 3628 - 3640, Jul 2015.

[24] M. Alodeh, S. Chatzinotas, and B. Ottersten, "Constructive multiuser interference in symbol level precoding for the MISO downlink channel," Signal Processing, IEEE Transactions on, vol. 63, no. 9, pp. 2239-2252, May 2015.

[25] G.S. Smith, "A direct derivation of a single-antenna reciprocity relation for the time domain," Antennas and Propagation, IEEE Transactions on, vol. 52, no. 6, pp. 1568-1577, 2004.

[26] M. Grant, S. Boyd, and Y. Ye, "CVX: Matlab software for disciplined convex programming," Online accessiable: http://stanford. edu $\sim$ boyd/cvx, 2008.

[27] Y. Huang and D.P. Palomar, "Rank-constrained separable semidefinite programming with applications to optimal beamforming," IEEE Transactions on Signal Processing, vol. 58, no. 2, pp. 664-678, Feb. 2010. 